\title{
DISCUSIONES
}

\section{SOBRE EL CONCEPTO DE REVOLUCIÓN CIENTÍFICA}

La publicación de las Exploraciones metacientíficas, de Ulises Moulines," constituye un verdadero acontecimiento, no sólo por ser uno de los pocos libros de filosofía de la ciencia escritos originalmente en castellano, sino también por la seriedad y la originalidad de los pensamientos que en él se exponen.

En este libro se desarrollan diversos temas que se encuentran conectados de alguna manera con la llamada "filosofía estructural de la ciencia"; dichos temas los agrupa Moulines en tres secciones. En la primera sección, que es la más larga, Moulines propone una serie de conceptos teóricos pertenecientes a la filosofía de la ciencia y, también, muestra el poder interpretativo que éstos tienen por medio de una serie de ejemplos de reconstrucciones de teorias científicas concretas en que se utilizan dichos conceptos. En la segunda sección, Moulines nos ofrece una serie de análisis historiográficos de diversas teorías científicas, sobre todo de la mecánica newtoniana. Cabe destacar que en esta sección no siempre se utiliza el instrumental estructural antes desarrollado; así, por ejemplo, en "Los fundamentos metodológicos de la filosofía natural de Isaac Newton", Moulines examina el empirismo de Newton, concluyendo que éste es de un tipo tal, que hace inteligible la mecánicà espiritualista y teleológica que Newton desarrolló en sus Principios; muy probablemente, en este artículo no se utiliza el instrumental estructural porque, aunque éste permita la reconstrucción de la teoría mecánica de Newton, no permite comprender los supuestos metafísicos y epistemológicos que animan a dicha teoría. Finalmente, en la ter-

* Alianza Editorial, Madrid, 1982. 
cera sección Moulines examina las implicaciones ontológicas de las teorías científicas.

Como he señalado, la filosofía de la ciencia que se desarrolla en este libro se inscribe dentro de la corriente estructural y, por ello, su propósito es desarrollar una metateoría de corte formalista que permita el análisis de la estructura de las teorías científicas. Ciertamente, la filosofía estructural que desarrolla Moulines es mucho más fina, y por ello más fructífera, que la filosofía de la ciencia desarrollada por el positivismo lógico. Así, por ejemplo, me parece muy atractiva, por realista, la sustitución de la noción de teoría como un sistema axiomático en el sentido clásico, por la de teoría como "red teórica arbórea", en que las leyes se relacionan, entre sí no sólo deductivamente sino también como "especializaciones" de leyes más generales. Por otra parte, pienso que el uso de un instrumento formal en la filosofía no es valioso porque sea formal y por ello supuestamente preciso, sino que sólo tiene sentido en la medida en que permite expresar con mayor exactitud ideas intuitivas interesantes y que no provienen de la misma lógica o de la matemática. En esto me parece que radica uno de los mayores aciertos de la obra de Moulines: en efecto, lo que encontramos en los capítulos dedicados al desarrollo de la metateoría estructural, no son una serie de formulismos que uno no entiende de dónde surgen y qué pretenden lograr, sino que Moulines nos presenta, generalmente a través del examen de casos concretos, las ideas intuitivas que fundamentan tal o cual propuesta formal metateórica. O sea que, a pesar de ser de corte formalista, la obra de Moulines es rica en ideas, las cuales, además, están presentadas en una forma asequible para un público que desconozca las sutilezas de los lenguajes lógicos.

Con respecto a la metateoría estructural desarrollada en este libro, considero que, si bien parece ser muy adecuada para el análisis sincrónico, y específicamente para lidiar con las llamadas "revoluciones científicas", cuya existencia Moulines mismo afirma, pienso que para que dicha metateoría sea capaz de dar cuenta de las revoluciones científicas, es necesario que amplíe su objeto de estudio, considerando que lo que se 
ha de analizar no son "tcorías centíficas" sino la "actividad científica".

Para justificar esta tesis, comenzaré con un breve examen del artículo "Forma y contenid de las revoluciones científicas: el casa de la mecánica newtoniana". En este artículo, Moulines nos dice que las revoluciones científicas consisten en cambios conceptuales profundos, o sea, en la alteración de los conceptos téenicos fundamentales de una disciplina o, en términos metateóricos, en la sustitución del núcleo básico de una red teórica por otro. Utilizando esta concepción de revolución cientffica, sostiene que la revokución newtoniana consistió en la spstitución de la teoría cartesiana por la teoría dinámica newtoniana (p. 255). Esta, ciertamente, no es la concepción usual del significado de la revolución newtoniana. Por ejemple, Kuhn, en su libro La revolución copernicana, sostiene que el significado de la obra newtoniana radica en ser la culminación de un proceso revolucionario gradual en que lo que lentamente se fue sustituyendo es el paradigma ptolomeico-aristotélico por el de la mecánica newtoniana.

Considero que el sentido kuhriano de revolución -desarrollado en La revolución copernicana- es preferible al de Moulines por las siguientes razones.

Moutines da dos argumentos específicos para sostener que la teoría newtoniana sustituye a la cartesiana y no a la aristotélica; a saber:

a) A pesar de formar parte de los estudios impartidos en las universidades, la teoría aristotélica no formaba parte de las discusiones de los físicos profesionales (p. 256). Esta afirmación me resulta un tanto asombrosa, porque no veo cuál podría ser un criterio aceptable para distinguir a los físicos "profesionales" del sigło XVII de los físicos universitarios, y considerar que sólo los físicos "profesionales" hacían una física seria o científica.

b) Moulines nos recuerda que Newton no dio argumentos específicos en contra de la teoría aristotélica, y sí en contra de la teoría cartesiana. Esto es cierto, y también es cierto que la teoría newtoniana sustituye a la cartesiana, pero esto no implica que nosotros no podamos afirmar que la importancia 
profunda de la obra newtoniana radica, no en haber desbancado a la mecánica cartesiana, que después de todo no llevaba más de 50 años de existencia, y que tampoco era la única -pues también existía una física o proyecto de física leibniziana-, sino en haber roto definitivamente con la larga tradición aristotélica, o sea, en haber roto con una manera de entender en qué consiste la realidad y en qué consiste el conocimiento de ésta. En otros términos, la ruptura radical, y por ello más significativa, que representa la obra newtoniana, no se localiza en el nivel de la sustitución de una teoría mecánica por otra, sino en el nivel, más profundo, de haber cambiado un modelo de conocimiento, y de la realidad, por otro modelo. Ahora bien, me parece que este sentido de revolución, como cambio no sólo de esquemas conceptuales, sino, más profundamente, como cambio de visión del mundo y de concepción de qué es conocer, sí es capturado por el concepto kuhniano de revolución, pero no por el de Moulines - y digo que Moulines no lo captura, precisamente porque limita la importancia de la obra newtoniana al mero hecho de haber sustituido a la mecánica cartesiana. Antes de desarrollar más este punto, quisiera dar algunas otras razones por las cuales el concepto kuhniano de revolución me parece preferible al de Moulines.

En primer lugar, asociada a la idea kuhniana de revolución como cambio profundo de visión del mundo y de concepción del conocimiento, está la idea de que las revoluciones son procesos graduales, dada la profundidad del cambio. Considero que es más coherente suponer que lo que ocurrió en los siglos XVI y XVII, entre Copérnico y Newton, fue un proceso revolucionario gradual, y no tan sólo un agregado de cuatro o más revoluciones, que es a lo que nos lleva la concepción de Moulines, ya que al restringirnos al plano de comparación de teorías, no es posible conceptuar cuál pueda ser el contenido o significado global de un proceso revolucionario gradual.

En segundo lugar, por una razón técnica, pienso que la sustitución de la teoría cartesiana por la newtoniana no puede, ni remotamente, considerarse como una revolución, porque la sustitución es parcial y no total, como requiere Moulines. En efecto, si bien es cierto que, como afirma Moulines, la ley 
fundamental 'newtoniana, $F=m a$, sustituye a la ley fundamental cartesiana del choque, no hay que olvidar que Descartes tiene otra ley fundamental, la ley de la inercia, que también está presente en la obra newtoniana y, más aún, como el primer axioma de la mecánica newtoniana. Ahora bien, el hecho de que la ley de inercia pueda subsumirse bajo la ecuación dinámica newtoniana, no implica que dicha ley no esté presente en la teoría cartesiana. Así pues, la sustitución de la teoría cartesiana por la newtoniana es parcial, porque si bien son teorías que difieren en cuanto a sus leyes dinámicas fundamentales, coinciden en un punto más fundamental, a saber, ambas son físicas inerciales, o sea, en ambas se afirma que lo único que hay que explicar apelando a fuerzas externas son los movimientos no-inerciales, o sea, los movimientos acelerados. Dado que la sustitución es parcial, ciertamente no estamos frente a un caso de revolución. Así pues, el cambio entre la teoría cartesiana y la newtoniana, no es una revolución en el sentido kuhniano de un cambio profundo, sino que ni siquiera es una revolución en el sentido mouliniano de la sustitución de una red teórica por otra. (Entre paréntesis, me parece que el otro supuesto ejemplo de revolución que analiza Moulines- el caso de la sustitución o, técnicamente, de la reducción aproximativa de la teoría kepleriana a la newtonianatampoco es una revolución ya que, brevemente, no tiene un esquema conceptual distinto al newtoniano: en efecto, la reformulación de las leyes de Kepler en términos de la magnitud "Kepler-teórica" no me parece una reformulación legítima, porque introduce un concepto teórico, mientras que en las leyes originales de Kepler no había ningún concepto Keplerteórico, sino sólo los no-teóricos de órbitas, elipses, áreas, tiempos, período. Por ello, considero que el caso Kepler-Newton no es un caso de reducción, y por tanto de revolución, sino un caso de teorización.)

Hemos estado argumentando que, frente al concepto mouliniano de revolución como sustitución de teorías, el concepto kuhniano de revolución, como un cambio profundo de visión del mundo y de modelo de conocimiento, resulta más plausible e interesante. Ahora bien, considero que lo que per- 
mite a Kuhn conceptuar la revolución como un cambio profundo es que concibe a la ciencia no meramente como teoría (en contraposición a la metateoría estructural), sino como teoría (i.e. leyes y aplicaciones paradigmáticas) más compromisos metafísicos y valores. Por compromisos metafísicos no hay que entender, tan sólo, el conjunto de los referentes de las leyes (como lo hace Moulines, p. 257), sino el significado que tienen estos referentes dentro de un contexto más amplio, usualmente filosófico. Así, por ejemplo, un compromiso metafísico fundamental de la teoría aristotélica es el de que el universo está constituído por substancias pertenecientes a distintas clases, en virtud de su esencia; en oposición a este compromiso fundamental aristotélico, el compromiso básico, tanto de Descartes como de Newton, es con la existencia de entidades no caracterizables por medio de esencias, sino concebidas homogénea y cuantitativamente, o sea, el mundo deja de estar poblado de elementos (tierra, agua), plantas, gatos, humanos, etc., y pasa a quedar poblado tan sólo por materia con o sin movimiento y, a veces, por espacio, tiempo y fuerzas, entidades que no difieren entre sí, ni son explicables en términos de esencias pero, en cambio, sí son caracterizables en términos cuantitativos. Si bien es cierto, como afirma Moulines en su artículo "Los fundamentos metodológicos de la filosofía natural de Isaac Newton", que Newton enfrentó al mecanicismo cartesiano una metafísica espiritualista, sosteniendo que aparte de la materia existía un espacio vacío y fuerzas entendidas como principios activos espirituales, cabe destacar que ésta es una discusión dentro de la misma tradición metafísica, en tanto que las nuevas entidades que Newton postula, también son caracterizables cuantitativamente, y no en términos de esencias. Asociados a estos compromisos metafísicos, hay ciertos valores explicativos, i.e., ciertos modelos de conocimiento. Conocer, para la tradición aristotélica, consiste en dar explicaciones cualitativas, en términos de esencias, mientras que para la tradición de la física clásiea conocer es dar explicaciones matematizad as de los fenómenos.

A partir de lo visto hasta aquí podemos concluir que:

1) El caso de la revolución newtoniana muestra que las re- 
voluciones científicas no se pueden concebir como meras sustituciones de teorías, como sostiene Moulines, ya que así concebidas resultaría que la obra newtoniana no es revolucionaria, ni con respecto a la cartesiana, ni con respecto a la kepleriana, además de que no se captura la profundidad o radicalidad de una revolución. (Entre paréntesis, dado que las revoluciones no pueden conceptuarse como meras sustituciones de teorías, creo que la noción metateórica de reducción, que Moulines desarrolla en los capítulos 2.8 y 2.9 , aunque es muy interesante en tanto trata de dar cuenta de cómo una teoría puede conservar el poder explicativo que tenía otra teoría, a pesar de tener un esquema conceptual distinto, no es una noción útil para conceptuar las revoluciones científicas, como sugiere Moulines.)

2) Una noción adecuada de revolución científica requiere, como hemos visto en el caso de la revolución newtoniana, que no sólo cambien las teorías, sino también los valores y los compromisos metafísicos. Por ello considero que, para que la metateoría estructural pueda servir para llevar a cabo análisis diacrónicos de la ciencia, y en particular de las revoluciones científicas, es necesario que dicha metateoría conciba que su objeto de estudio no son teorías científicas, sino tradiciones científicas (o sea, leyes junto con sus aplicaciones paradigmáticas, o modelos, más compromisos metafísicos y valores).

Ahora bien, la introducción de compromisos científicos y valores dentro del objeto de estudio de la metateoría estructural, conllevaría otra gran ventaja, a saber, nos permitiría explicar la génesis de teorías científicas. Como Moulines dice, correctamente: "Sin la admisión de este principio [activo, espiritual y no-mecánico]. . . Newton no habría concebido la teoría matemática de la gravitación" (p. 275, subrayado mío); o sea, lo que motivó el desarrollo newtoniano de una teoría de fuerzas de acción a distancia, fue su adhesión a la metafisica neoplatonista de Cambridge. Ciertamente creo que una metateoría que permitiese el análisis, no sólo del contenido de las teorías cientificas, sino también de las razones intra y ex trateóricas (como, por ejemplo, la metafísica neoplatonista) que explican el desarrollo de una teoría, scría una metateoría 
muy rica. $\mathrm{Y}$ dado que la metateoría estructural ya ha roto con varios supuestos y prejuicios del positivismo lógico, como son los de la concepción enunciativa de las teorías y la dicotomía normativo-descriptiva, creo que tal vez podría, y ciertamente debería, romper con la limitativa dicotomía contexto de justificación/contexto de descubrimiento, planteando que el objeto del análisis filosófico no es sólo el contenido de las teorías, sino también su génesis y desarrollo. Me parece que una manera de lograr esto es introduciendo compromisos metafísicos y valores en el concepto de red teórica.

Para terminar, quisiera destacar lo siguiente. Moulines sostiene que lo que es realmente esencial para entender la naturaleza y evolución de las teorías científicas es el contenido de las teorías, y por lo cual la tarea del filósofo de la ciencia consiste tan sólo en descubrir las estructuras conceptuales de las teorías (pp. 52-3). Respecto de este planteamiento básico, que anima a toda la metateoría estructural, difiero por dos razones. En primer lugar, como he in tentado mostrar arriba, un análisis diacrónico adecuado, que permita dar cuenta tanto de las revoluciones científicas como de la génesis y desarrollo de teorías, requiere que se conciba que el objeto de estudio de la filosofía no es el análisis del contenido conceptual de las teorías, sino el análisis de las tradiciones científicas (i.e., teorías, más compromisos metafísicos, más valores). En segundo lugar, la evolución de las teorías no sólo está condicionada por problemas técnicos internos a la ciencia, como sostiene Moulines, sino también por "factores externos", i.e., socioeconómicos, políticos, ideológicos y psicológicos. Aunque éste no es el lugar apropiado para argumentar en favor de esta tesis, mencionaré un ejemplo: creo que la única manera de poder explicar por qué el dar cuenta del movimiento planetario fue uno de los proyectos fundamentales de los participantes de la revolución científica de los siglos XVI y XVII es apelando a factores socioeconómicos, tales como la necesidad de reforma del calendario, la necesidad económica de mejorar las técnicas de navegación, etc. Así pues, dado que el desarrollo histórico de la ciencia no puede explicarse realmente sin apelar a factores externos, considero que la metateoría estructural debería in- 
cluirlos también dentro de su objeto de estudio, o sea, considerar que lo que ha de analizarse es la actividad científica, o sea, teorías, compromisos metafísicos y valores que son producidos y utilizados socialmente (y por socialmente entiendo no sólo comunidades científicas, sino a las sociedades en su conjunto). Esta propuesta difiere de la de Moulines en tanto que, para él, los factores externos son estudiados por otras disciplinas distintas a la filosofía de la ciencia, aunque guardan algún tipo de conexiones entre sí; en cambio, en la propuesta que he hecho se considera que dichos factores deben incluirse en el objeto mismo de estudio de la filosofía de la ciencia. O sea, estas propuestas difieren, no en considerar a los factores externos pertinentes o no en la explicación del desarrollo científico, sino en considerarlos esenciales o no.

En síntesis, pienso que para que la metateoría estructural pueda servir para hacer análisis diacrónicos de la ciencia es necesario que conciba que su objeto de estudio no son meramente las teorías científicas, sino la actividad científica. 